\title{
Motional Stark effect imaging first results on the DIII-D tokamak
}

Cite as: Rev. Sci. Instrum. 89, 10D124 (2018); https://doi.org/10.1063/1.5038969

Submitted: 07 May 2018 . Accepted: 19 June 2018 . Published Online: 08 October 2018

Alex Thorman (D, Clive Michael (D), John Howard (D), Brian Victor, Chris Holcomb, and Steve Allen
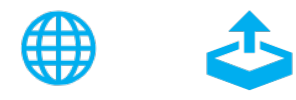

\section{ARTICLES YOU MAY BE INTERESTED IN}

Laser calibration of the DIII-D coherence imaging system

Review of Scientific Instruments 89, 10E110 (2018); https://doi.org/10.1063/1.5038739

Application of the VUV and the soft x-ray systems on JET for the study of intrinsic impurity behavior in neon seeded hybrid discharges

Review of Scientific Instruments 89, 10D131 (2018); https://doi.org/10.1063/1.5038930

Coherence-imaging spectroscopy for 2D distribution of ion temperature and flow velocity in a laboratory magnetosphere

Review of Scientific Instruments 89, 10D133 (2018); https://doi.org/10.1063/1.5037124

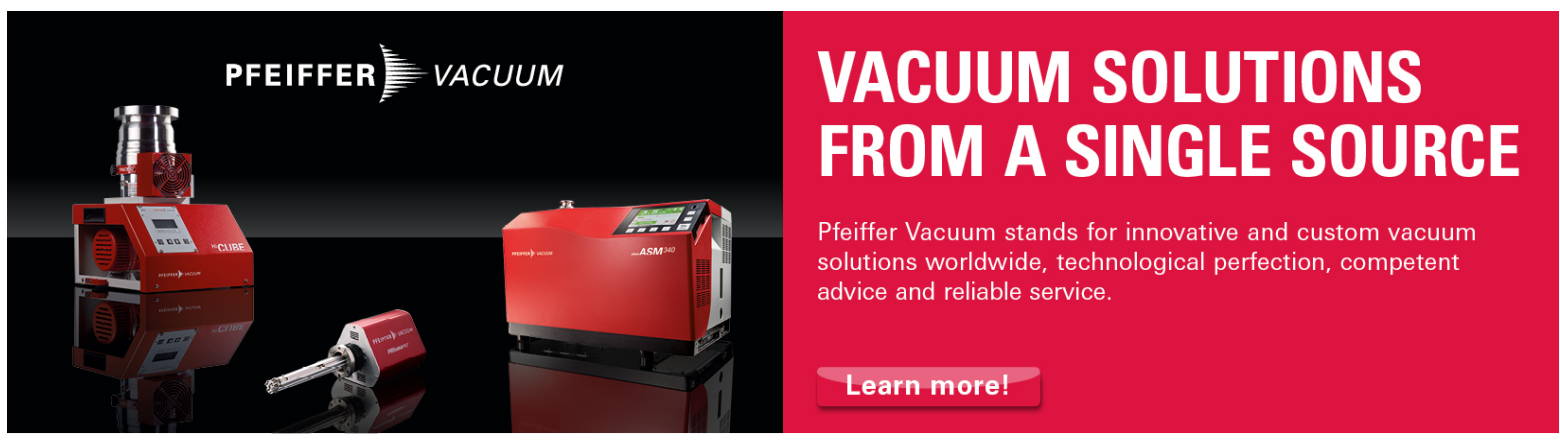




\title{
Motional Stark effect imaging first results on the DIII-D tokamak
}

\author{
Alex Thorman, ${ }^{1}$ Clive Michael, ${ }^{1}$ John Howard,${ }^{1}$ Brian Victor,${ }^{2}$ Chris Holcomb, ${ }^{2}$ and Steve Allen ${ }^{2}$ \\ ${ }^{1}$ Plasma Research Laboratory, Research School of Physics and Engineering, Australian National University, \\ Canberra, ACT 0200, Australia \\ ${ }^{2}$ Lawrence Livermore National Laboratory, Livermore, California 94550, USA
}

(Presented 17 April 2018; received 7 May 2018; accepted 19 June 2018; published online 8 October 2018)

\begin{abstract}
A motional Stark effect (MSE) imaging diagnostic was benchmarked against existing conventional MSE polarimeters on the DIII-D tokamak and delivered new capabilities for measuring the magnetic pitch angle from 2 neutral beams and on the high field side of DIII-D. Line integration across flux surfaces was considerable for the radial view utilised; nevertheless, the imaging MSE measurements from both beams were self-consistent and in close agreement with conventional MSE measurements. The ferroelectric liquid crystal waveplate used in the imaging polarimeter was discovered to have spatially non-uniform retardance; hence, it is necessary for the calibration source to replicate the ray paths of the neutral beam emission through the optical system. Published by AIP Publishing. https://doi.org/10.1063/1.5038969
\end{abstract}

\section{INTRODUCTION}

Motional Stark effect (MSE) polarimetry is a widely used spectroscopic diagnostic for constraining the toroidal current profile in tokamaks. ${ }^{1}$ The diagnostic observes Doppler shifted Balmer-alpha emission from neutral deuterium atoms injected into the plasma. The atoms experience a motional electric field $\mathbf{E}_{\mathbf{L}}=\mathbf{v} \times \mathbf{B}$ in their rest frame that splits the emission line, via the Stark effect, into $\pi$ and $\sigma$ components which are polarised parallel and perpendicular to the electric field, respectively. Measurement of the polarisation angle reveals the direction of the electric field and the "pitch" of the magnetic field. Conventional MSE polarimeters use narrowband filters to isolate either a $\sigma$ or $\pi$ component for polarisation analysis, requiring an individual filter for each radial viewing position as the Doppler shift varies across the field of view of the beam. This typically limits the diagnostic to having 10s of channels in the midplane of the device. Conventional MSE polarimeters have been used on the DIII-D tokamak for over 25 years ${ }^{2,3}$ and have since been expanded to 69 channels viewing two different beams from four different directions. ${ }^{4}$

A more recently developed MSE polarimetry technique utilises birefringent crystals to effectively establish a sinusoidal spectral filter instead of the usual narrowband filters. The technique does not require the Doppler shift to be precisely tracked across the field of view and therefore permits imaging of the entire neutral beam emission on a camera sensor. ${ }^{5}$ The increased resolution and 2D nature of imaging MSE (IMSE) have opened new opportunities for directly measuring non-inductive current drive, ${ }^{6}$ instabilities, ${ }^{7}$ and edge pedestal structures. ${ }^{8}$ A temporally switched IMSE polarimeter ${ }^{5}$ was installed on DIII-D for a two month campaign in 2016 for first time benchmarking of IMSE against

Note: Paper published as part of the Proceedings of the 22nd Topical Conference on High-Temperature Plasma Diagnostics, San Diego, California, April 2018. conventional MSE and for measurements on the high field side of the plasma. This particular IMSE encoding technique has previously been implemented on the TEXTOR ${ }^{9,10}$ and $\mathrm{KSTAR}^{6,8,11}$ tokamaks and differs from the fully spatial encoding technique favoured on the ASDEX-U tokamak. ${ }^{5,7,12}$ The IMSE viewing geometry on DIII-D, design considerations for the polarimeter, and calibration procedure are considered in this paper in Secs. II-IV, respectively. Results highlighting the capabilities of the IMSE diagnostic are shown in Sec. V, including benchmarking against conventional MSE measurements and complications relating to line integration. Improvements for future implementations of the system are discussed in the Conclusion.

\section{VIEWING GEOMETRY}

The IMSE polarimeter was installed on the " $15^{\circ}$ MSE" port, shown in Fig. 1, that has previously been used for dualview measurements of both the radial and motional electric fields with conventional MSE polarimeters. ${ }^{13}$ The port was chosen after considering port availability and the need for preexisting Faraday rotation calibrations, given the limited calibration options for the relatively short campaign. Favourably the port has a direct view of the plasma without any need for a mirror and includes two neutral beam sources with similar Doppler shifts, making high field side measurements possible. A $2 \mathrm{~nm}$ IMSE filter passband, centred on $\sim 661 \mathrm{~nm}$, transmits light from both beams and is tilted to partially track the Doppler shift variation across the field of view. Another upside is that the sightlines are predominantly parallel to the plasma radial electric field, and therefore its effect on the polarisation angle is small.

The radial resolution from the IMSE view is limited by line of sight integration across the $\delta R \sim 20 \mathrm{~cm}$ range of flux surfaces covered by the $\approx 14 \mathrm{~cm}$ wide neutral beam. ${ }^{13}$ Consequently each IMSE sightline averages over a significant range of polarisation angles, as shown in Fig. 2, where effects relating to the finite aperture, focusing, and divergence of the neutral 


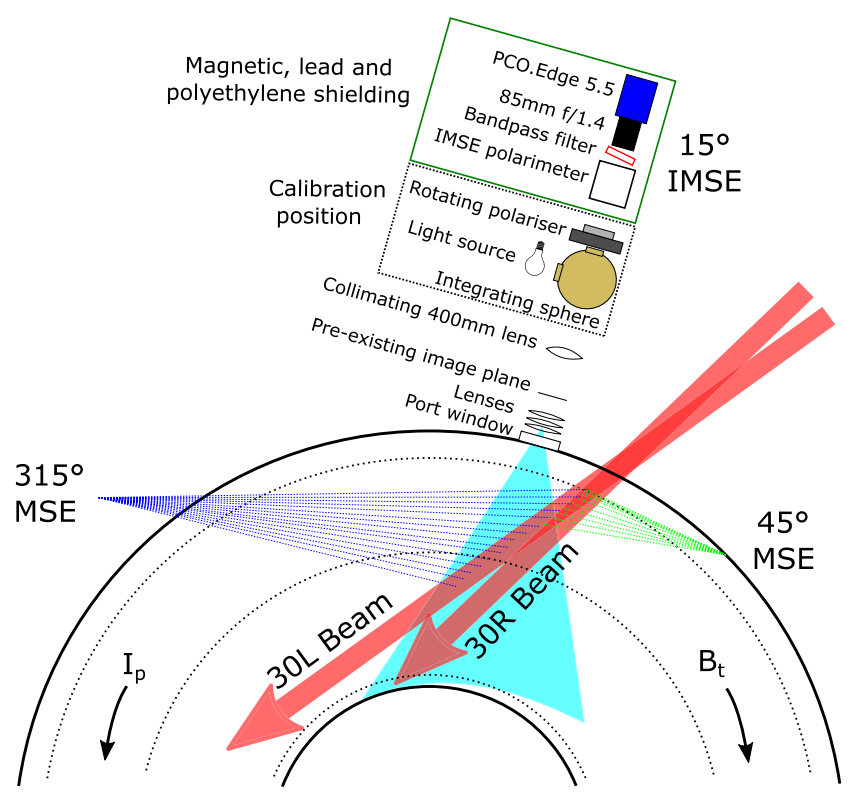

FIG. 1. Diagram of the IMSE optical assembly, viewing geometry, and relevant neutral beams. The conventional MSE systems view individual radial positions on the $30 \mathrm{~L}$ beam, while the imaging MSE system is operable with either the 30L or 30R beam. Curved dotted lines indicate the standard position of the inner and outer separatrices at the midplane and the magnetic axis. The image plane of the previous conventional MSE system is an intermediate image in the IMSE installation, and the $400 \mathrm{~mm}$ lens and further downstream optics are on an optical rail detached from the tokamak. The position and components only present when calibrating the polarimeter are indicated.

beam have been simulated, as well as the variation in magnetic field pitch angle across the flux surfaces. Near $R=1.8 \mathrm{~m}$, the IMSE sightline averages over an $\approx 20^{\circ}$ range of polarisation angles, significantly larger than the $<1^{\circ}$ range encountered at the tangential " $315^{\circ}$ MSE" view. The average polarisation angle for each IMSE sightline can be $>1^{\circ}$ from the simplistic prediction assuming an infinitely narrow non-diverging beam, an effect most evident near $R=1.5 \mathrm{~m}$ for the $30 \mathrm{R}$ beam.
It should be noted that the far side of the neutral beam-sightline intersection contributes the lower polarisation angles. Hence slightly greater average polarisation angles can be expected compared to that in Fig. 2 when neutral beam attenuation is also considered, given that the far sides of the beams enter the plasma earlier, propagate further through the plasma, and encounter higher density plasma than the beam on the near side sightline intersection.

\section{DIAGNOSTIC OVERVIEW}

The polarimeter layout for the temporally switched single spatial heterodyne system ${ }^{5}$ used for the IMSE measurements is shown in Fig. 3. The fast axis orientation of the "half-wave" ferroelectric liquid crystal (FLC) waveplate switches in between camera exposures to produce different weightings of the linear Stokes components. This idealised polarimeter is only sensitive to linearly polarised light, and the transmitted spectral intensity for each state of the FLC can be determined from a Mueller matrix analysis to be

$$
\begin{aligned}
& S_{45^{\circ}}=\frac{1}{2}\left(s_{0}+s_{1} \sin \phi+s_{2} \cos \phi\right), \\
& S_{90^{\circ}}=\frac{1}{2}\left(s_{0}+s_{1} \sin \phi-s_{2} \cos \phi\right) .
\end{aligned}
$$

For the IMSE measurement, the modulating element, known as a displacer, is a uniaxial birefringent crystal with the optic axis cut at an angle $\Theta$ to the surface of the crystal. The displacer produces a large offset delay as well as a delay shear dependent on the incident angle of the light, with the exact form presented in Ref. 14. In the focal plane of a lens, these dominant components of the delay are given by

$$
\begin{gathered}
\phi(\omega, x, y)=\phi_{A}(\omega)+\phi_{B}(\omega, x, y), \\
\phi_{A}=\frac{\omega L n_{o}}{c}\left(1-\frac{n_{e}}{\sqrt{n_{e}^{2} \sin ^{2} \Theta+n_{o}^{2} \cos ^{2} \Theta}}\right),
\end{gathered}
$$
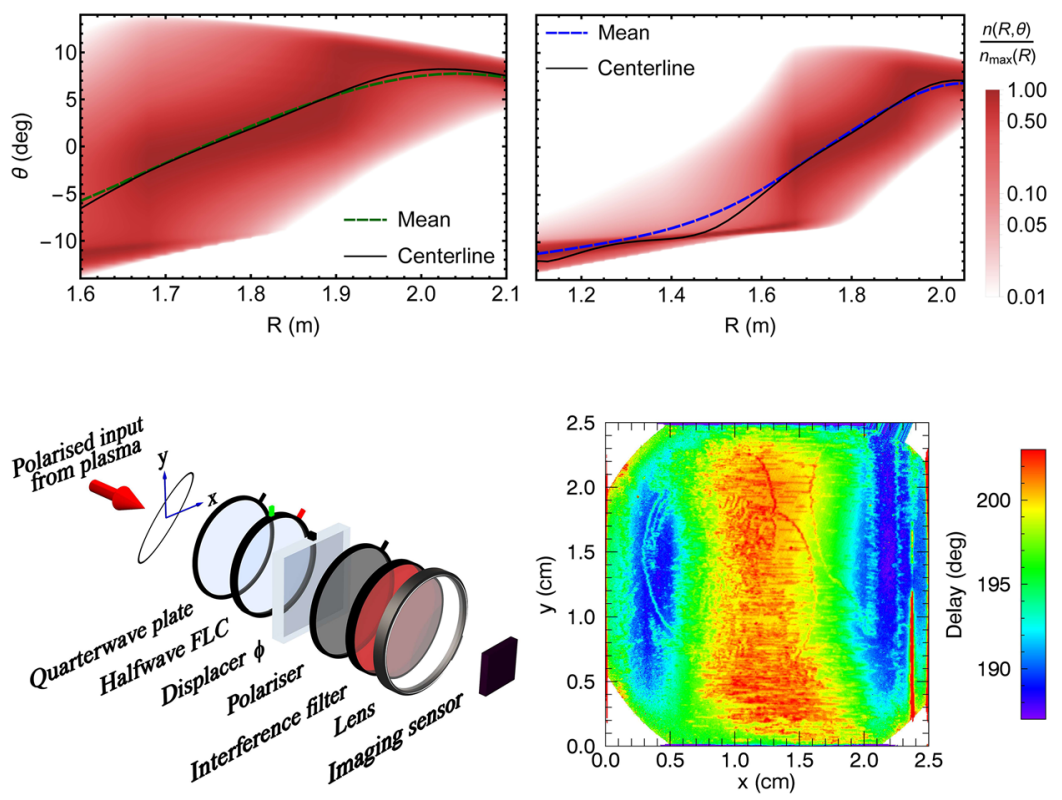

FIG. 2. (Left) Simulated distribution of 30L polarisation angles $\theta$ for lines of sight intersecting the beam centerline at different radii $R$ on the midplane. The dashed green line is the weighted mean angle of the distribution, while the black line represents the polarisation angle at the beam centerline. (Right) Polarisation angles for the 30R beam, with a greater radial range than $30 \mathrm{~L}$ due to the different beam geometries.
FIG. 3. (Left) Layout of the temporally switched IMSE polarimeter. The displacer crystal provides the interferometric delay $\phi$ and spatially modulated carrier fringes. The FLC fast axis switches between $45^{\circ}$ and $90^{\circ}$ to establish a differential measurement from the carrier fringe phase. The notches indicate the orientation of the waveplate fast axes. (Right) Measured spatial dependence of the FLC retardance. 


$$
\phi_{B} \approx \frac{2 \omega L \Delta n \sin 2 \Theta}{\left(n_{e}+n_{o}\right) c f} y=k_{y} y,
$$

where $n_{e}(\omega)$ and $n_{o}(\omega)$ are the extraordinary and ordinary refractive indices, $\Delta n=n_{e}-n_{o}, L$ is the crystal thickness, $c$ is the speed of light, and $f$ is the focal length of the lens. The light angular frequency $\omega$ is used for convenience, and second order $\Delta n, x$, and $y$ terms have been dropped in Eq. (5). The spatial carrier $k_{y}$ in the image plane delivers a signal across a range of Doppler shifts. Additionally the dispersion of $\phi(\omega)$ is exploited to establish a sinusoidal spectral filter [ $\sin \phi$ and $\cos \phi$ in Eqs. (1) and (2)] over the MSE spectrum to constructively interfere the orthogonally polarised $\sigma$ and $\pi$ components. For the relatively narrow emission, a linear approximation of the dispersion is applicable and is given by

$$
\left.\left.\frac{\partial \phi}{\partial \omega}\right|_{\omega=\omega_{0}} \approx\left(1+\frac{\omega}{\Delta n} \frac{\partial \Delta n}{\partial \omega}\right) \frac{\phi}{\omega}\right|_{\omega=\omega_{0}}=\kappa \frac{\phi\left(\omega_{0}\right)}{\omega_{0}},
$$

where $\omega_{0}$ is the unshifted angular frequency of the MSE emission. When the spectral periodicity of the sinusoidal filter roughly matches the spacing of the $\pi$ wings, a large net signal is obtained. Consequently the signal contrast is maximised by choosing a displacer that approximately satisfies ${ }^{15}$

$$
\phi_{A}\left(\omega_{0}\right)=\frac{2 \pi \omega_{0} \hbar}{9 \kappa e a_{0}|\mathbf{v} \times \mathbf{B}|},
$$

where $\hbar$ is the reduced Planck constant, $e$ is the elementary charge, and $a_{0}$ is the Bohr radius.

Integrated over the MSE spectrum, the signal measured by the sensor for the two FLC states is

$$
\begin{aligned}
& I_{45^{\circ}}=\frac{I_{0}}{2}\left(1+\zeta \sin \left[\phi_{0}(y)+\alpha+2 \theta_{\sigma}\right]\right), \\
& I_{90^{\circ}}=\frac{I_{0}}{2}\left(1+\zeta \sin \left[\phi_{0}(y)+\alpha-2 \theta_{\sigma}\right]\right),
\end{aligned}
$$

where $I_{0}$ is the total intensity and $\phi_{0}(y)=\phi\left(\omega_{0}, 0, y\right)$ is the carrier wave given in Eqs. (3)-(5). Evidently the desired polarisation angle of the $\sigma$ component $\theta_{\sigma}$ is encoded in the phase difference between the carrier waves in the $45^{\circ}$ and $90^{\circ} \mathrm{FLC}$ states. The fringe contrast $\zeta$ and phase offset $\alpha$ are described in detail elsewhere. ${ }^{5}$ Spatial variations in $I_{0}, \zeta, \alpha$, and $\theta_{\sigma}$ are all expected to vary gradually relative to the carrier $\phi_{0}(y)$ and therefore only contribute to a broadening of the spatial carrier $k_{y}$.

The displacer thickness $L$ and optic axis cut angle $\Theta$ that provide the maximum signal contrast and desired fringe frequency can be determined from Eqs. (4), (5), and (7). For the DIII-D system, a $5 \mathrm{~mm} \alpha \mathrm{BBO}$ displacer $\left(n_{e}=1.549\right.$, $n_{o}=1.666$, and $\kappa=1.07$ at $660 \mathrm{~nm}^{16}$ ) with cut angle $\Theta=30^{\circ}$ generates a delay of $\phi_{A}=684$ waves and a fringe frequency of $k_{y}=3.5 \times 10^{4}=\mathrm{rad} \mathrm{m}^{-1}$ or 27 pixels per fringe $(85 \mathrm{~mm}$ lens focused onto $6.5 \mu \mathrm{m} \times 6.5 \mu \mathrm{m}$ camera pixels). To increase the vertical spatial resolution in the image, the fringe frequency could be increased to $\sim 10$ pixels per fringe, without significantly degrading the fringe contrast. The interference fringes of the IMSE polarimeter were oriented to give the maximal spatial resolution in the radial direction; however, in reality, the radial resolution from the port was limited by line of sight integration highlighted in Fig. 2.

\section{A. Non-ideal FLC retardance}

Non-ideal components in the polarimeter will introduce $s_{3}$ and further $s_{1}$ and $s_{2}$ polarised components into the weighting of the $\sin \phi$ and $\cos \phi$ carriers in Eqs. (1) and (2). Notably, the Stark-Zeeman circular polarisation is weak for the IMSE view as the sightlines are largely perpendicular to the magnetic field; hence, $s_{3}$ can be neglected. The greatest concern is the non-ideal FLC retardance (Fig. 3) which is larger than halfwave and spatially non-uniform. In this case, the polarimeter response generalises to ${ }^{11}$

$$
\begin{aligned}
& I_{45^{\circ}}=\frac{I_{0}}{2}\left(1+\zeta_{1} \sin \left[\phi_{0}(y)+\alpha+2 \theta_{\sigma}+4 g\right]\right), \\
& I_{90^{\circ}}=\frac{I_{0}}{2}\left(1+\zeta_{2} \sin \left[\phi_{0}(y)+\alpha-2 \theta_{\sigma}+4 h\right]\right)
\end{aligned}
$$

for some functions $g\left(\theta_{\sigma}\right), h\left(\theta_{\sigma}\right), \zeta_{1}\left(\theta_{\sigma}\right)$, and $\zeta_{2}\left(\theta_{\sigma}\right)$. The variation of the contrast terms $\zeta_{1}$ and $\zeta_{2}$ is typically small and only important to the measurement through signal to noise effects. With a non-ideal FLC retardance of $\pi+\beta$, the phase correction functions are

$$
\begin{gathered}
g(\theta)=\frac{1}{16} \sin (4 \theta) \beta^{2}+O\left(\beta^{4}\right), \\
h(\theta)=\beta / 4 .
\end{gathered}
$$

Evidently a non-ideal FLC introduces a $\beta / 4$ offset to the ideal phase difference encoding of the polarisation angle along with a weak variation with the input linear polarisation angle. The exact form of $g(\theta)$ and $h(\theta)$ can be calibrated experimentally or modelled when the characteristics of the entire system are known. ${ }^{11}$

\section{CALIBRATION}

Faraday rotation measurements through the port window and front end optics were available at 10 radial locations from the previously installed conventional MSE system. The $0.4^{\circ} \mathrm{T}^{-1}$ variation across the field of view was extrapolated to the IMSE view under the assumption that the toroidal field is the dominant influence. The vertical dependence of the Faraday rotation and possible rotation from the additional lenses in the IMSE installation are unknown but expected to be small.

The response of the polarimeter [i.e., the functions $g$ and $h$ in Eqs. (10) and (11)] was calibrated using a bright white light source that fills an integration sphere before passing through a linear polariser on a digitally controlled rotation stage, as indicated in Fig. 1. A $0.3 \mathrm{~nm}$ wide, $660 \mathrm{~nm}$ passband filter is used as a proxy for the neutral beam light source. A calibration offset of $g(0)-h(0) \approx-3.3^{\circ}$ was measured for the response which is consistent with the non-ideal $\sim 195^{\circ}$ FLC retardance (Fig. 3) effects expected from Eq. (13). Higher order $\theta_{\sigma}$ corrections are also evident and can be calibrated.

To verify the integrity of this calibration procedure, a linear polariser was inserted in front of the polarimeter during a plasma shot. Before the FLC retardance non-uniformity had been realised, it was expected that the test would give the same results as the calibration light source. However the neutral beam illumination measurement was different, as 


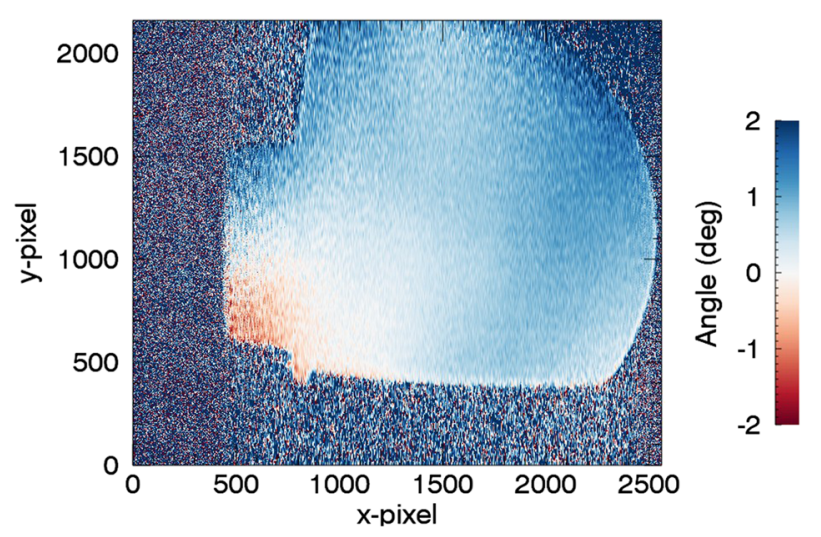

FIG. 4. Difference in the polarimeter response for a horizontal polariser illuminated with the neutral beam relative to the integration sphere. The outline of the viewing port is evident in the image.

seen in Fig. 4 where the measured value of $(g-h)_{\text {plasma }}-$ $(g-h)_{\text {sphere }}$ is displayed. The difference arises because the effective delay imparted by the FLC is dependent on the range of ray paths through it. While the FLC retardance varies by $15^{\circ}$ spatially, the ray path weighted effective retardance in the imaging system will have a smaller range. As per Eqs. (12) and (13), a $\pm 4^{\circ}$ variation in the effective FLC retardance can give rise to the observed $\approx \pm 1^{\circ}$ differences between the plasma and calibration illumination. Comparisons between the neutral beam and calibration light were obtained at three different polariser orientations $\left(\theta \approx 0^{\circ}, \pm 10^{\circ}\right)$, and the relative changes were found to be $<0.2^{\circ}$, in agreement with Eq. (12). Therefore this neutral beam measurement is applied as a fixed offset to the integration sphere calibration but has some residual uncertainties.

\section{RESULTS}

A comparison of the midplane pitch angles measured with IMSE from both beams and conventional MSE is shown in Fig. 5 for a shot with interleaved $30 \mathrm{~L}$ and $30 \mathrm{R}$ beam timing.

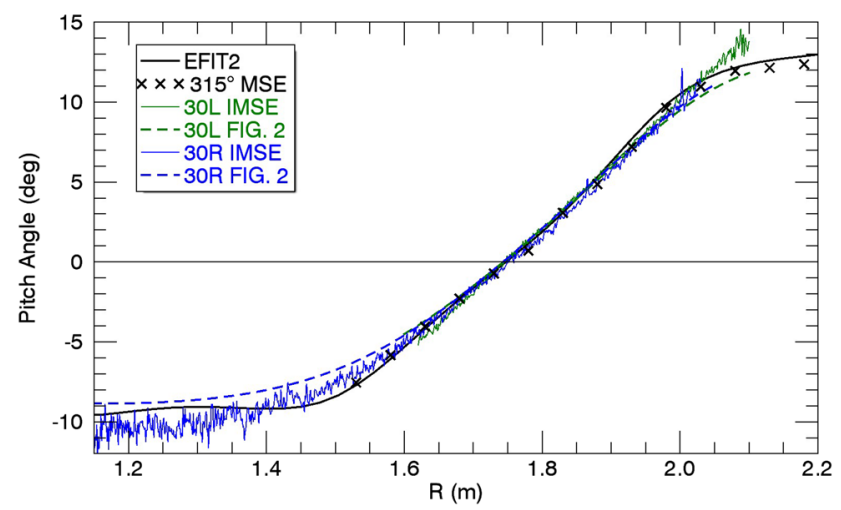

FIG. 5. Pitch angles measured on the midplane from 30L and 30R IMSE, $315^{\circ} \mathrm{MSE}$, and conventional MSE constrained EFIT equilibrium reconstruction for shot 166599 at $t \approx 2.2 \mathrm{~s}$. A conversion from the polarisation angle to the pitch angle has been applied to account for the different beam and viewing geometries under the assumption $B_{R}=0$ and $E_{r}=0$. Line integration corrections are not applied to the IMSE data, but the dashed lines indicate the expected offsets simulated in Fig. 2.
The line integration effects, simulated for the same shot and time in Fig. 2, are essential for assessing differences between the measurements. Line integration was predicted to cause $1^{\circ}(10 \%)$ larger than "expected" polarisation angle near $R=1.5 \mathrm{~m}$ from the $30 \mathrm{R}$ beam, an effect partially replicated in Fig. 5. In the region $R<1.4 \mathrm{~m}$, the offset is in the opposite direction to Fig. 2 and cannot be explained by beam attenuation effects which were anticipated to shift the weighting of the line integral to larger polarisation angles. However it should be noted that the beam attenuation significantly reduces the signal in this region; hence, the measurement is more susceptible to systematic errors. Furthermore radial electric fields and changes in the current profile during the $40 \mathrm{~ms}$ spaced beam blips may contribute to this difference. The 30L IMSE measurements are also in close agreement with the line integrated simulation. The most notable difference occurs above $R>2$ m where the IMSE filter only transmits a fraction $(<30 \%)$ of the multiplet as the Doppler shift decreases, leading to a weak signal.

The IMSE data can be reduced to a number of individual channels to compare directly with conventional MSE measurements. Figure 6 presents a comparison of the pitch angle temporal evolution measured by the conventional MSE and IMSE with a $33 \mathrm{~Hz}$ camera frame rate. Line integration effects are particularly evident for the $R=2.03 \mathrm{~m}$ channel for the $\mathrm{L}$ to $\mathrm{H}$-mode transition occurring at $3 \mathrm{~s}$. For this channel, the pitch angle measured by the conventional MSE, which has a radial resolution better than $5 \mathrm{~cm},{ }^{13}$ remains approximately constant, while the pitch angle at $R=1.98 \mathrm{~m}$ drops $\approx 1^{\circ}$ and at $R=1.93 \mathrm{~m}$ drops $\approx 2^{\circ}$. Effectively there is an increase in the toroidal current near $R=1.98 \mathrm{~m}$ at $3 \mathrm{~s}$. Meanwhile the $R=2.03$ m IMSE "channel" has radial resolution of only $\approx 16 \mathrm{~cm}$ and is therefore sensitive to this drop in the pitch angle occurring for $R<2 \mathrm{~m}$ unlike the conventional MSE view.

The information contained in the vertical $Z$ direction in an IMSE image has the potential to further constrain the shape of the magnetic flux surfaces. A comparison of the IMSE measured polarisation angle with a MSE midplane constrained

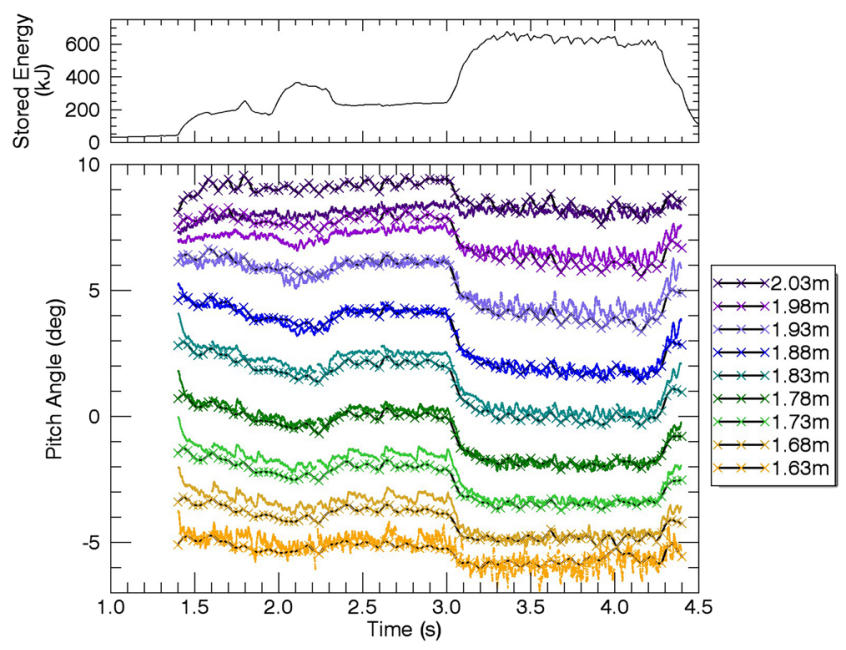

FIG. 6. Comparison of IMSE (crosses connected with black lines) and $315^{\circ}$ conventional MSE (coloured lines) measured pitch angles for shot 165748. 


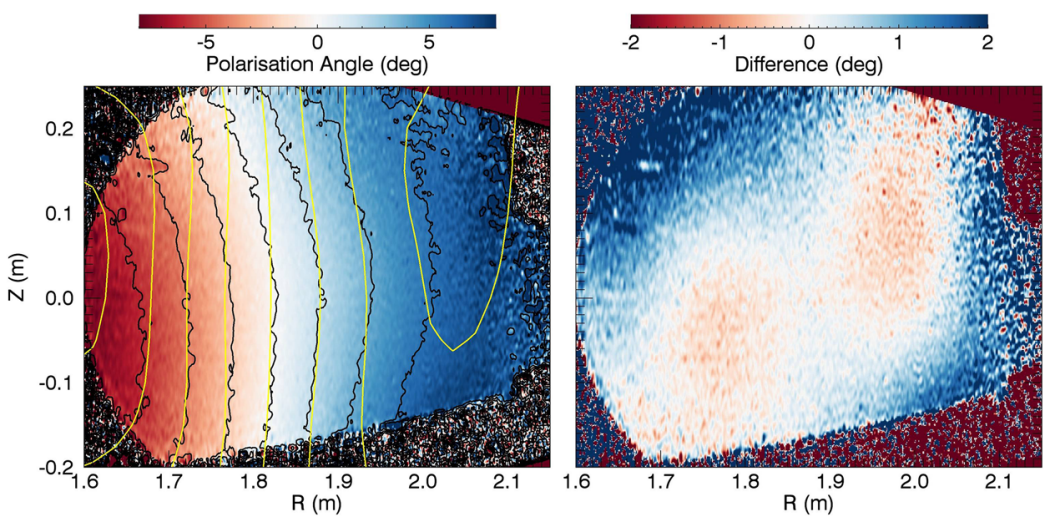

FIG. 7. (Left) 30L IMSE polarisation angle measured at $3.5 \mathrm{~s}$ in shot 165748 . Forward modelled conventional MSE constrained EFIT contours are shown in yellow. (Right) Difference between the IMSE measurement and the EFIT forward modelled polarisation angle assuming a narrow beam with horizontal velocity.
EFIT is given in Fig. 7. Close agreement is evident near the midplane, as anticipated from Figs. 5 and 6; however, $\sim 1^{\circ}$ differences exist above and below the midplane. Although EFIT is not constrained off the midplane, discrepancies are not anticipated to be this significant. The IMSE contours of constant polarisation angle appear to fan slightly outwards from the bottom of the image, and this artefact was observed for most shots in the IMSE campaign. The possibility for this artefact to result from line integration or the vertical divergence of the neutral beam velocity has been considered and discounted. It is suggested that some systematic errors exist in the IMSE data resulting from the extrapolation Faraday rotation that does not include the additional optics in the IMSE system or possibly the aperture of the calibration polariser used for the correction in Fig. 4 was not sufficiently large to completely replicate the neutral beam illumination.

\section{CONCLUSION}

The utility of IMSE was highlighted by its ability to also measure the MSE signal from the 30R beam, providing first time measurements on the high field side of the plasma. The IMSE measurements were complicated by the limited radial resolution inherent to the view; however, close agreement is demonstrated between measurements from both beams and with the conventional MSE. Some residual uncertainties still exist in the IMSE calibration related to the extrapolation of the Faraday rotation and the FLC illumination dependence. More accurate calibrations with the temporally switched IMSE system in the future could utilise a large aperture linear polariser that is gradually rotated in front of the IMSE polarimeter across the relevant range of angles during a plasma shot, as implemented on ASDEX-U. ${ }^{12}$ In-vessel or beam into gas calibration is the preferred but less readily available option. Furthermore obtaining a more ideal spatially uniform FLC or an alternative time switching waveplate is considered to be imperative.

\section{ACKNOWLEDGMENTS}

The authors would like to thank Robert and Helen Crompton, and AINSE Ltd for providing financial assistance. This work was supported in part by the U.S. Department of Energy, Office of Science, Office of Fusion Energy Sciences, using the DIII-D National Fusion Facility, a DOE Office of Science user facility, under Award Nos. DE-FC02-04ER54698 and DE-AC52-07NA27344.

This report was prepared as an account of work sponsored by an agency of the United States Government. Neither the United States Government nor any agency thereof, nor any of their employees, makes any warranty, express or implied, or assumes any legal liability or responsibility for the accuracy, completeness, or usefulness of any information, apparatus, product, or process disclosed, or represents that its use would not infringe privately owned rights. Reference herein to any specific commercial product, process, or service by trade name, trademark, manufacturer, or otherwise, does not necessarily constitute or imply its endorsement, recommendation, or favoring by the United States Government or any agency thereof. The views and opinions of authors expressed herein do not necessarily state or reflect those of the United States Government or any agency thereof.

${ }^{1}$ F. M. Levinton, R. J. Fonck, G. M. Gammel, R. Kaita, H. W. Kugel, E. T. Powell, and D. W. Roberts, Phys. Rev. Lett. 63, 2060 (1989).

${ }^{2}$ D. Wróblewski, K. H. Burrell, L. L. Lao, P. Politzer, and W. P. West, Rev. Sci. Instrum. 61, 3552 (1990).

${ }^{3}$ D. Wróblewski and L. L. Lao, Rev. Sci. Instrum. 63, 5140 (1992).

${ }^{4}$ C. T. Holcomb, M. A. Makowski, R. J. Jayakumar, S. A. Allen, R. M. Ellis, R. Geer, D. Behne, K. L. Morris, L. G. Seppala, and J. M. Moller, Rev. Sci. Instrum. 77, 10E506 (2006).

${ }^{5}$ J. Howard, Plasma Phys. Controlled Fusion 50, 125003 (2008).

${ }^{6}$ C. A. Michael, A. Thorman, J. Chung, Y. Bae, J. H. Jeong, J. Decker, and J. Howard, in 42nd European Physical Society Conference on Plasma Physics, EPS 2015, Presented at the 42nd EPS Conference on Plasma Physics (EPS, Lisbon, 2015), Vol. 65, p. 2014.

${ }^{7}$ O. P. Ford, A. Burckhart, R. McDermott, T. Pitterich, and R. C. Wolf, Rev. Sci. Instrum. 87, 11E537 (2016).

${ }^{8}$ J. Howard, C. Michael, H. Chen, R. Lester, A. Thorman, and J. Chung, J. Instrum. 10, P09023 (2015).

${ }^{9}$ J. Howard, J. Phys. B: At., Mol. Opt. Phys. 43, 144010 (2010).

${ }^{10}$ J. Howard, A. Diallo, R. Jaspers, and J. Chung, Plasma Fusion Res. 5, S1010 (2010).

${ }^{11}$ A. Thorman, C. Michael, and J. Howard, Rev. Sci. Instrum. 84, 063507 (2013).

${ }^{12}$ O. P. Ford, J. Howard, and R. C. Wolf, Rev. Sci. Instrum. 86, 093504 (2015).

${ }^{13}$ B. W. Rice, K. H. Burrell, L. L. Lao, and Y. R. Lin-Liu, Phys. Rev. Lett. 79, 2694 (1997).

${ }^{14}$ F. E. Veiras, L. I. Perez, and M. T. Garea, Appl. Opt. 49, 2769 (2010).

${ }^{15}$ A. Thorman, C. Michael, M. De Bock, and J. Howard, Rev. Sci. Instrum. 87, 073504 (2016).

${ }^{16}$ K. Kato, IEEE J. Quantum Electron. 22, 1013 (1986). 